\title{
Machine-learning identification of galaxies in the WISE $\times$ SuperCOSMOS all-sky catalogue ${ }^{\star}$
}

\author{
T. Krakowski ${ }^{1}$, K. Małek ${ }^{1,2}$, M. Bilicki ${ }^{3,2}$, A. Pollo ${ }^{1,4,2}$, A. Kurcz ${ }^{4,2}$, and M. Krupa ${ }^{4,2}$ \\ ${ }^{1}$ National Centre for Nuclear Research, ul. Andrzeja Sołtana 7, 05-400 Otwock, Poland \\ e-mail: tomasz.krakowski@ncbj.gov.pl \\ 2 Janusz Gil Institute of Astronomy, University of Zielona Góra, ul. Lubuska 2, 65-265 Zielona Góra, Poland \\ 3 Leiden Observatory, Leiden University, PO Box 9513, 2300 RA Leiden, The Netherlands \\ 4 Astronomical Observatory of the Jagiellonian University, ul. Orla 171, 30-244 Kraków, Poland
}

Received 22 June 2016 / Accepted 11 August 2016

\begin{abstract}
Context. The two currently largest all-sky photometric datasets, WISE and SuperCOSMOS, have been recently cross-matched to construct a novel photometric redshift catalogue on $70 \%$ of the sky. Galaxies were separated from stars and quasars through colour cuts, which may leave imperfections because different source types may overlap in colour space.

Aims. The aim of the present work is to identify galaxies in the WISE $\times$ SuperCOSMOS catalogue through an alternative approach of machine learning. This allows us to define more complex separations in the multi-colour space than is possible with simple colour cuts, and should provide a more reliable source classification.

Methods. For the automatised classification we used the support vector machines (SVM) learning algorithm and employed SDSS spectroscopic sources that we cross-matched with WISE $\times$ SuperCOSMOS to construct the training and verification set. We performed a number of tests to examine the behaviour of the classifier (completeness, purity, and accuracy) as a function of source apparent magnitude and Galactic latitude. We then applied the classifier to the full-sky data and analysed the resulting catalogue of candidate galaxies. We also compared the resulting dataset with the one obtained through colour cuts.

Results. The tests indicate very high accuracy, completeness, and purity ( $>95 \%)$ of the classifier at the bright end; this deteriorates for the faintest sources, but still retains acceptable levels of $\sim 85 \%$. No significant variation in the classification quality with Galactic latitude is observed. When we applied the classifier to all-sky WISE $\times$ SuperCOSMOS data, we found 15 million galaxies after masking problematic areas. The resulting sample is purer than the one produced by applying colour cuts, at the price of a lower completeness across the sky.

Conclusions. The automatic classification is a successful alternative approach to colour cuts for defining a reliable galaxy sample. The identifications we obtained are included in the public release of the WISE $\times$ SuperCOSMOS galaxy catalogue.
\end{abstract}

Key words. methods: data analysis - methods: numerical - astronomical databases: miscellaneous - galaxies: statistics large-scale structure of Universe

\section{Introduction}

Modern wide-field astronomical surveys include millions of sources, and future catalogues will increase these numbers to billions. As most of the detected objects cannot be followed-up spectroscopically, research done with such datasets will heavily rely on photometric information. Without spectroscopy, an appropriate identification of various source types is complicated, however. In the seemingly most trivial case of star-galaxy separation in deep-imaging catalogues, we quickly reach the limit where this cannot be done based on morphology: we lack resolution, and distant faint galaxies become unresolved or pointlike, similar to stars (e.g. Vasconcellos et al. 2011). Additional information is then needed to separate out these and sometimes other classes of sources (such as point-like but extragalactic quasars). This has traditionally been done with magnitude and colour cuts; however, when the parameter space is multidimensional, such cuts become very complex. Additionally, noise in photometry scatters sources from their true positions in

$\star$ The public release of the WISE $\times$ SuperCOSMOS galaxy catalogue is available from http://ssa.roe.ac.uk/WISExSCOS the colour space. This, together with huge numbers of sources, most of which are usually close to the survey detection limit, precludes reliable overall identification with any manual or byeye methods. For these reasons, the idea of automatized source classification has recently gained popularity and was applied to multi-wavelength datasets such as AKARI (Solarz et al. 2012), the Panoramic Survey Telescope and Rapid Response System (Pan-STARRS, Saglia et al. 2012), the VIMOS Public Extragalactic Redshift Survey (VIPERS, Małek et al. 2013), the crossmatch of the Wide-field Infrared Survey Explorer - Two Micron All Sky Survey (WISE-2MASS; Kovács \& Szapudi 2015), the Sloan Digital Sky Survey (SDSS, Brescia et al. 2015), and the WISE-only (Kurcz et al. 2016), and it has been tested in view of the Dark Energy Survey data (Soumagnac et al. 2015).

The present paper describes an application of a machinelearning algorithm to identify galaxies in a newly compiled dataset, based on the two currently largest all-sky photometric catalogues: WISE in the mid-infrared, and SuperCOSMOS in the optical. This work is a refinement of a simpler approach at source classification that was applied in Bilicki et al. (2016), hereafter B16, where stars and quasars were filtered out on a statistical basis using colour cuts to obtain a clean galaxy sample for 
the purpose of calculating photometric redshifts. The two parent catalogues we use here, described in detail below and in Sect. 2, both include about a billion detections each, of which a large part are in common. For various reasons, however, the available data products from these two surveys offer limited information on the nature of the catalogued objects, which indeed presents a challenge to the classification task.

WISE (Wright et al. 2010), which is the more sensitive of the two, suffers from low native angular resolution resulting from the small aperture of the telescope $(40 \mathrm{~cm})$ : it is equal to $6.1^{\prime \prime}$ in its shortest $W 1$ band $(3.4 \mu \mathrm{m})$, increasing to $12^{\prime \prime}$ at the longest wavelength $W 4$ of $23 \mu \mathrm{m}$. This leads to severe blending in crowded fields, such as at low Galactic latitudes, and original photometric properties of the blended sources become mixed. In addition, proper isophotal photometry has not been performed for the majority of WISE detections, and no WISE all-sky extended source catalogue is available as yet (see, however, Cluver et al. 2014; and Jarrett et al. 2016, for descriptions of ongoing efforts to improve on this situation). Finally, WISEbased colours provide limited information for classification purposes: at rest frame, the light in its two most sensitive passbands, $W 1$ and $W 2$ (3.4 and $4.6 \mu \mathrm{m})$, is emitted from the photospheres of evolved stars (Rayleigh-Jeans tail of the spectrum), and the catalogue is dominated by stars and galaxies of relatively low redshift, which typically have similar $W 1-W 2$ colours. The two other WISE filters, W3 and W4 centred on 12 and $23 \mu \mathrm{m}$, respectively which might serve to reliably separate out stars from galaxies and QSOs when combined with $W 1$ and $W 2$ (Wright et al. 2010), offer far too low detection rates to be applicable for most of the WISE sources.

SuperCOSMOS (hereafter SCOS) on the other hand, which is based on the scans of twentieth century photographic plates (Hambly et al. 2001c), does offer point and resolved source identification (Hambly et al. 2001b). This classification, although quite sophisticated, is based mostly on morphological information, however, and on the one hand, unresolved galaxies and quasars are classified as point sources, and on the other, blending in crowded fields (Galactic Plane and Bulge, Magellanic Clouds) leads to spurious extended source identifications (see also Peacock et al. 2016).

A cross-match of the WISE and SCOS catalogues improves the classification of different types of sources that is useful for extragalactic applications, as shown in B16. However, although only extended SCOS sources were considered in B16, blends mimicking resolved objects dominated at Galactic latitudes as high as $\pm 30^{\circ}$ and had to be removed on a statistical basis. In the present paper we improve on that work by generating a wide-angle (almost full-sky) galaxy catalogue from the WISE $\times$ SCOS cross-match through machine-learning. For this purpose we use the support vector machines (SVM) supervised algorithm.

A similar task for other WISE-based datasets was undertaken in two recent works. Kovács \& Szapudi (2015), who used a cross-match of WISE $W 1<15.2$ sources with the 2MASS Point Source Catalogue (PSC, Skrutskie et al. 2006) and performed an SVM analysis in multicolour space, showed that a cut in the $W 1_{\text {WISE }}-J_{2 \text { MASS }}$ colour efficiently separates stars and galaxies. Based on these results, they produced a galaxy catalogue containing 2.4 million objects with an estimated star contamination of $1.2 \%$ and a galaxy completeness of $70 \%$. The separation was only made for stars and galaxies, with no information regarding quasars. A limitation of a WISE - 2MASS cross-match is the much smaller depth of the latter with respect to the former. Most of the 2MASS galaxies are located within $z<0.2$ (Bilicki et al. 2014; Rahman et al. 2016), while WISE extends well beyond this, detecting $L_{*}$ galaxies at $z \sim 0.5$ (Jarrett et al. 2016).

Using only photometric information from WISE, Kurcz et al. (2016) employed SVM and attempted classifying all unconfused WISE sources brighter than $W 1<16$ into three classes: stars, galaxies, and quasars. This led to identification of 220 million candidate stars, 45 million candidate galaxies, and 6 million candidate QSOs. The latter sample is however, significantly contaminated with what was interpreted as a possibly very local foreground, such as asteroids or zodiacal light.

The present paper is laid out as follows: the data are described in Sect. 2, Sect. 3 explains the principles of the support vector machine-learning algorithm and introduces the training sample used here, and in Sect. 4 we present various tests that allowed us to quantify the performance of the SVM algorithm. Section 5 contains the description and properties of the final galaxy catalogue, as well as a comparison with the results of B16. In Sect. 6 we summarise our analysis.

\section{Data: WISE and SuperCOSMOS}

The catalogues used to construct the main photometric dataset, WISE and SuperCOSMOS, are comprehensively described in Bilicki et al. (2014) and B16. Here we briefly summarize them and the preselections applied for the purpose of this project. They are practically equivalent to those from the latter paper; for more details see Sect. 2 of B16, and in particular Table 1 and Figs. 1-3 therein.

\subsection{WISE}

The Wide-field Infrared Survey Explorer (WISE; Wright et al. 2010), a NASA space-based mission, surveyed the entire sky in four mid-infrared (IR) bands: 3.4, 4.6, 12, and $23 \mu \mathrm{m}$ (W1 $W 4$, respectively). Here we used its second full-sky release, the AllWISE dataset ${ }^{1}$ (Cutri et al. 2013), combining data from the cryogenic and post-cryogenic survey phases. It includes almost 750 million sources with signal-to-noise $(\mathrm{S} / \mathrm{N})$ ratio $\geq 5$ in at least one of the bands, and its averaged $95 \%$ completeness in unconfused areas is $W 1 \lesssim 17.1, W 2 \lesssim 15.7, W 3 \lesssim 11.5$, and $W 4 \lesssim 7.7$ in Vega magnitudes, with variable coverage, however, that is highest at ecliptic poles and lowest near the ecliptic, especially in stripes resulting from Moon avoidance manoeuvres.

Our WISE preselection required sources with $\mathrm{S} / \mathrm{N}$ ratios higher than 2 in the $W 1$ and $W 2$ bands, and that they were not obvious artefacts (cc_flags $[1,2] \neq$ "DPHO”); this yielded 603 million detections over the whole sky. Owing to the low survey resolution $\left(\sim 6^{\prime \prime}\right)$, the immensely crowded Galactic Plane and Bulge are entirely dominated by stellar blends, and extracting particularly extragalactic information is practically impossible (extinction is not such a problem in the WISE passbands unless in very high-extinction regions, however). We therefore focused on the $83 \%$ of the sky available at $|b|>10^{\circ}$, which reduced the sample to about 460 million objects.

The depth of WISE observations is position dependent because of the scanning strategy, and it is highest at the ecliptic poles (Jarrett et al. 2011) and lowest at the ecliptic ${ }^{2}$. The

\footnotetext{
1 Available for download from IRSA at http://irsa.ipac. caltech.edu

2 http://wise2.ipac.caltech.edu/docs/release/allwise/ expsup/sec2_2.html
} 
95\% completeness limit of AllWISE over large swaths of unconfused sky is $W 1=17.1 \mathrm{mag}(\mathrm{Vega})^{3}$, and we independently verified by analysing the WISE source distribution in W1 magnitude bins that adopting a flux limit of $W 1<17$ leads to a relatively uniform selection as far as instrumentally driven artefacts are concerned. This cut gave a final input WISE dataset of about 343 million sources at $|b|>10^{\circ}$. At the bright end, this sample is dominated by stars even at high Galactic latitudes (Jarrett et al. 2011, 2016), and we estimate about 100 million of the WISE sources, mostly faint, to be galaxies and quasars (B16; Kurcz et al. 2016), the remainder are of stellar nature.

The WISE database currently does not offer reliable (e.g. isophotal) aperture photometry for the resolved sources and they are not even identified therein (except for fewer than 500000 cross-matches with the 2MASS Extended Source Catalogue). We therefore used the w?mpro magnitudes (where the question mark stands for the channel number), which are based on point spread function profile-fit measurements. The only proxy for morphological properties that we adopted here from the database is given by circular aperture measurements performed on the sources within a series of fixed radii. These were obtained without any contamination removal or compensation for missing pixels, however. In particular, as in Bilicki et al. (2014) and Kurcz et al. (2016), in the classification procedure we used a differential measure (a concentration parameter), that is defined as

w1mag13 =w1mag_1 - w1mag_3,

where w1mag_1 and w1mag_3 were measured in fixed circular apertures of radii of $5.5^{\prime \prime}$ and $11^{\prime \prime}$, respectively. The w1mag13 parameter is expected to have different distributions for point and resolved sources, which indeed is the case, as we verified against SDSS spectroscopic data described in Sect. 3.1.

We note that of the four available WISE bands we did not employ the longest wavelength $W 4$ because of its very low sensitivity, which leads to an overwhelming number of nondetections. In addition, whenever $W 3$ was used, all the sources with w3snr $<2$ (upper limits and non-detections, which together dominate the $W 3$ channel in our sample), were artificially dimmed by +0.75 mag to statistically compensate for their overestimated fluxes, which we determined to be an appropriate average correction (see Appendix A for details). Possible errors associated with this procedure are not important for our final catalogue, however, as we finally did not employ the $12 \mu \mathrm{m}$ passband for the overall classification because of the photometry issues, although it does bring some improvement (cf. Sect. 4.1). The W3 information was used only in the test phase.

\subsection{SuperCOSMOS}

The SuperCOSMOS Sky Survey (SCOS, Hambly et al. 2001a,b,c) consists of digitized photographs in three bands, $B, R, I$, obtained through automated scanning of source plates from the United Kingdom Schmidt Telescope (UKST) in the south and the Palomar Observatory Sky Survey-II (POSS-II) in the north. The observations were conducted in the last decades of the twentieth century. The data are publicly available from the SuperCOSMOS Science Archive ${ }^{4}$, with photometric, morphological, and quality information for 1.9 billion sources.

\footnotetext{
3 http://wise2.ipac.caltech.edu/docs/release/allwise/ expsup/sec2_4a.html

4 http://surveys.roe.ac.uk/ssa/
}

SCOS provides source classification flags in each of the three bands, as well as a combined one, meanclass, which is equal to 1 if the source is resolved, 2 if it is unresolved, 3 if it is unclassifiable, and 4 if it is likely noise (Hambly et al. 2001b), the two latter cases comprising a negligible fraction $(\ll 1 \%)$ of all the sources. The derived catalogue of extended sources was accurately calibrated all-sky using SDSS photometry in the relevant areas, and the calibration was extended over the remaining sky by matching plate overlaps and by using the average colour between the optical and 2MASS $J$ bands (Peacock et al. 2016). This was not the case for the point sources, however, which very much limits their applicability for uniform source selection. In B16 only the SCOS sources with meanClass $=1$ were used to obtain a WISE $\times$ SCOS galaxy sample that was further scourged of residual quasars and stars. In the present paper we followed this preselection, but we recall that only a part of these SCOS sources are in fact extragalactic. Especially at low Galactic latitudes, this extended source catalogue is dominated by blends of stars with other stars and with extragalactic objects. The remaining SCOS preselections are also the same as in B16 and earlier in Bilicki et al. (2014): objects need to be properly detected with aperture photometry in the $B$ and $R$ bands $^{5}$ (gCorMagB and gCorMagR2 not null; quality flags qualB and qualR2 $<2048$, meaning no strong warnings or severe defects, Hambly et al. 2001b).

The publicly available catalogue was supplemented with additional data in corners of the photographic plates that were missing from the original dataset because of so-called stepwedges (Hambly et al. 2001b). This mostly affects low declinations. The $B$ and $R$ magnitudes were additionally calibrated between the north and the south (the split being at $\delta_{1950}=2.5^{\circ}$ ) to compensate for differences between effective passbands of UKST and POSS-II; see Peacock et al. (2016) for details.

To preserve the all-sky photometric reliability and to mitigate problems with catalogue depth that varies from plate to plate, two flux limits were applied to the SCOS dataset, $B<21$ and $R<19.5$ (AB-like, extinction corrected). As already mentioned, we did not use the Galactic Plane strip of $|b|<10^{\circ}$, where blending and high extinction make SCOS photometry unreliable. The resulting SCOS catalogue of extended sources outside of the Galactic Plane includes over 85 million objects.

\subsection{Cross-matched WISE $\times$ SuperCOSMOS photometric sample}

The two photometric catalogues were paired using a matching radius of $2^{\prime \prime}$. The resulting flux-limited cross-matched sample at $|b|>10^{\circ}$ contains almost 48 million sources. This number includes WISE sources supplemented from an earlier cryogenic phase of observations ("All-Sky", Cutri et al. 2012), to fill in strips of missing data centred on ecliptic longitudes of $\lambda \sim 50^{\circ}$ and $\lambda \sim 235^{\circ}$.

All the magnitudes were extinction corrected using the Schlegel et al. (1998) maps throughout and applying the following extinction coefficients, derived from the Schlafly \& Finkbeiner (2011) re-calibration (B16): $A_{B}=3.44$, $A_{R}=2.23, A_{W 1}=0.169$, and $A_{W 2}=0.130$. The usage of de-redenned magnitudes also for stars is motivated by the fact that we focus on extragalactic sources, the stellar ones being contamination for our applications. As these corrections are often significant in the optical, neglecting them would lead to considerable biases in the final galaxy catalogue. Still, we did

\footnotetext{
5 We did not use the third SCOS band, $I$, as it is too shallow.
} 

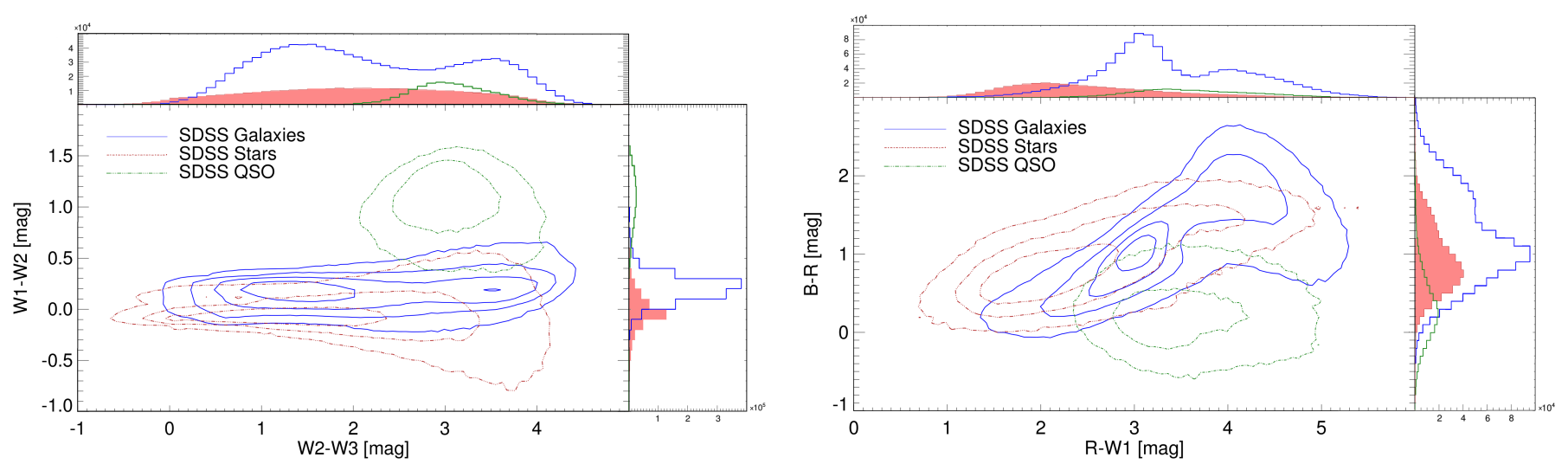

Fig. 1. Colour-colour diagrams for galaxies, stars, and quasars from a cross-match of WISE $\times$ SuperCOSMOS with SDSS spectroscopic data. Left panel: WISE colours only; right panel: WISE and SCOS colours. Blue contours correspond to galaxies, red contours represent stars, and green contours illustrate quasars.

not use the areas of very high extinction, $E(B-V)>0.25$, which have almost no training data for classification, and the photometry especially in the optical is problematic. This cut, removing another 7.2 million sources, is the same as applied in B16, where the appropriate threshold was determined through an analysis of spurious under- and overdensities in WISE $\times$ SCOS source distribution.

Figure 1 shows why using an automatic classifier rather than simple colour cuts is more suitable to separate galaxies from stars and quasars in the WISE $\times$ SCOS sample. The diagrams illustrate distributions of three source types (galaxies, quasars, and stars) on two colour-colour (c-c) planes. Source identifications come from the WISE $\times$ SCOS $\times$ SDSScross-match described in detail in Sect. 3.1. In the left panel we show the $W 2-W 3$ vs. $W 1-W 2$ c-c plane, which is often used for object separation in WISE (e.g. Jarrett et al. 2011; Ferraro et al. 2015). The plot shows that it is challenging to find simple cuts in these parameters that would maximise both the completeness and the purity of the resulting samples. While we might quite well separate QSOs from other sources, for example by a $W 1-W 2=0.8$ cut (Stern et al. 2012; Assef et al. 2013; Yan et al. 2013), it is much more difficult, if possible at all, to apply a single threshold to efficiently separate galaxies from stars. The galaxy and star distributions overlap very much even if the additional colour $W 2-W 3$ is taken into account. The situation is similar for other colour combinations that are available from the five bands in WISE $\times$ SCOS. The right panel of Fig. 1 illustrates the $R-W 1$ vs. $B-R \mathrm{c}-\mathrm{c}$ plane, where the three source types also largely overlap.

\section{Classification method: support vector machines}

For the classification performed in this work we used the SVM method. SVM is a supervised learning algorithm that is a maximum-margin classifier able to determine decision planes between sets of objects with different class memberships, to establish a decision boundary by maximising the margin between the closest points of the classes (the socalled support vectors). Each single object is classified based on its relative position in the $n$-dimensional parameter space (Cristianini \& Shawe-Taylor 2000; Shawe-Taylor \& Cristianini 2004; Solarz et al. 2012; Małek et al. 2013).

The SVM algorithm is an increasingly popular way of handling astronomical data to classify different types of objects. For various applications of SVM in astronomy we refer to, for instance, Woźniak et al. (2004), Huertas-Company et al. (2008), Solarz et al. (2012), Saglia et al. (2012), Małek et al. (2013), Kovács \& Szapudi (2015), Marton et al. (2016), and Kurcz et al. (2016).

In our case, SVM was used to build a non-linear classifier for the photometric data in the WISE $\times$ SCOS all-sky catalogue. As input data we used photometric information such as magnitudes, colours, and a differential aperture magnitude (see Sect. 4 for details). The input data were transformed by a kernel into a higher-dimensional feature space, where the separation between different classes is less complex than in the input parameter space. For more details see for example Manning et al. (2008) or Małek et al. (2013); an illustrative description of how SVM classification operates is provided in Han et al. (2016).

The SVM algorithm searches for a boundary $B$ in the feature space that will separate examples from different categories by maximizing a fitness function $F$ :

$F=M-C \sum_{i} \xi_{i}(B, M)$,

where $M$ is the margin of the boundary, and $\xi(B, M)$ is the number of training examples violating this criterion. The cost parameter $C$ is a trade-off between large margins and poor classification. Equation (2) shows that for very large $C$ each training example on the wrong side of the margin is heavily penalized. When $C$ is small, individual $\xi_{i}$ penalize Eq. (2) less heavily, thus the optimal boundary may be one that misclassfies a small number of outliers. More details can be found in Beaumont et al. (2011).

For our particular implementation, we used a C-SVM algorithm with a Gaussian kernel to identify three different classes of objects: galaxies, quasars, and stars, with the final aim to reliably pinpoint the galaxies. The Gaussian kernel function (also dubbed radial basic) is defined as

$k\left(x_{i}, y_{j}\right)=\exp \left(-\gamma\left\|x_{i}-x_{j}\right\|^{2}\right)$,

where $\left\|x_{i}-x_{j}\right\|$ is the Euclidean distance between feature vectors in the input space. The parameter $\gamma$ is related to the breadth of the Gaussian distribution, $\sigma$, namely $\gamma=1 /\left(2 \sigma^{2}\right)$, and determines the topology of the decision surface. Too high a value of $\gamma$ sets a complicated decision boundary, while too low $\gamma$ can give a decision surface that is too simple, which might cause misclassifications. The classifier is trained using a subset of input data for which class identifications are known. In our case, the training 
set was derived from SDSS DR12 spectroscopic data matched with WISE $\times$ SCOS (see Sect. 3.1).

The two parameters, $C$ and $\gamma$, were optimized based on the training set through a grid search and $N$-fold cross-validation; we used $N=10$, in which case the training data were split into ten equal sets, and the classifier was trained on nine of them. Then the classifier was tested against the remaining tenth subset (the so-called self-check or validation). This test was repeated ten times with a different subset removed for each training run. The classification accuracy was then calculated by averaging over the ten runs. The same method was used in Solarz et al. (2012) and Małek et al. (2013), where a more detailed description of this process is provided. Moreover, an additional test sample was used (data with known classification, but not used for training) for an independent check of the classifier performance.

In the test phase of our study, we used only the discrete classes assigned by SVM to each source. For the final classification, however, we decided to employ the full probability distributions for each class. This allowed us to examine the cases of problematic classification where the probabilities that a source belongs to two or three classes were roughly equal. This is discussed in detail in Sect. 5.

For our analysis we used LIBSVM6 (Chang \& Lin 2011), integrated software for support vector classification, which allows for multiclass identifications. We also employed $\mathrm{R}$, a free software environment for statistical computing and graphics, with the e1071 interface (Meyer 2001) package installed.

\subsection{Training sample: SDSS DR12 spectroscopic data}

A well-chosen training sample is crucial for the SVM method, because the classifier is tuned based on the properties of this sample: the $C$ and $\gamma$ parameters are estimated and the hyperplane between classes is determined. This means that a representative sample of sources, with known properties that we wish to identify, is essential. In our specific case of a catalogue including $z \lesssim 0.5$ galaxies (Bilicki et al. 2014; B16) as well as stars and higher-redshift quasars, such a training set requires good-quality and high-reliability pre-classification using spectroscopic measurements. For this reason, for training and testing purposes we chose to employ the spectroscopic sample from the Sloan Digital Sky Survey Data Release 12 (SDSS DR12, Alam et al. 2015) cross-matched with the WISE $\times$ SCOS dataset defined above.

The SDSS is a multi-filter imaging and spectroscopic survey, and its DR12, used for our analysis, includes dedicated star, galaxy, and quasar surveys. These samples are shallower than the imaging part of the SDSS, but they are available with high reliability only from spectra (Bolton et al. 2012): the photometric classification of SDSS was only based on source morphology (resolved vs. point-like, Stoughton et al. 2002). SDSS DR12 contains almost 3.9 million spectroscopic sources, of which $61 \%$ were identified as galaxies, $22 \%$ as stars, and the remaining $16 \%$ as quasars/AGNs (SDSS class “QSO"). To avoid unreliable spectroscopic measurements and hence problematic classification, we used additional information on the redshift from the SDSS database as a quality determinant: the zWarning flag and the relative error in redshift (radial velocity for stars) defined as $\Delta z=z_{\text {err }} / z$, where $z_{\text {err }}$ is the database value. Only the sources with zWarning $=0$ were used throughout, with the additional conditions of $\Delta z<0.1$ for galaxies and quasars, and $\Delta z<1$ for stars.

Pairing these sources with our WISE $\times$ SCOS flux-limited catalogue within $1^{\prime \prime}$ matching radius resulted in over 1 million common objects, $95 \%$ of which were galaxies, $2 \%$ were stars,

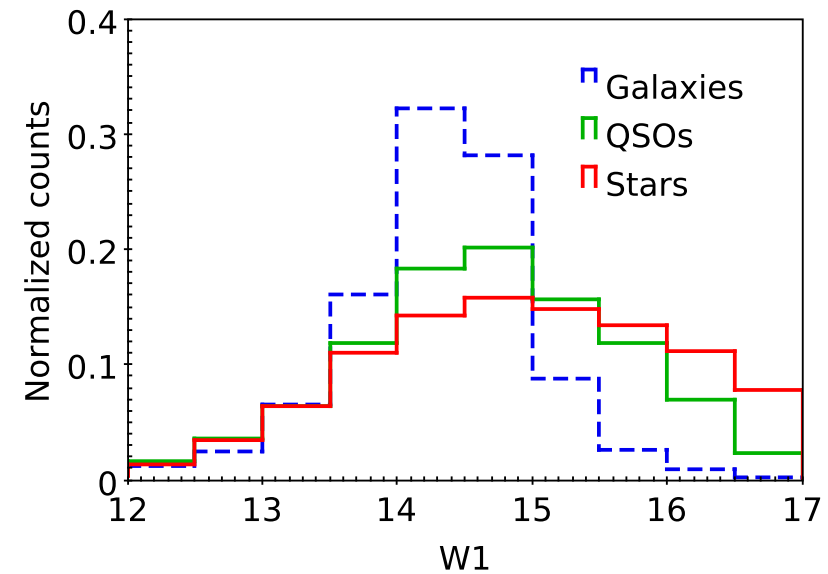

Fig. 2. Normalised number counts of $W 1$ magnitudes in the WISE $\times$ SCOS $\times$ SDSS cross-matched sample for stars, galaxies, and quasars.

and $3 \%$ were quasars. Clearly, the stars and most of the quasars are point sources and should not be resolved. That we identified over 50000 of them in the cross-match of SDSS with the WISE $\times$ SCOS extended source catalogue reflects the susceptibility of SCOS morphological classification (the meanClass flag) to blending, which mimics resolved sources (B16). The main purpose of the present study is to reliably filter out such sources from the galaxy catalogue we aim to produce.

\section{SVM classification performance}

This section describes various tests made using the SDSS-based training sample, which allowed us to quantify the performance of the SVM algorithm in view of the final classification of the entire catalogue.

To check the classification efficiency, to calculate the dependence on different parameters, and to perform the final classification, the following procedure was used: (1) as galaxy properties change with magnitude, each sample (training and test sets, final catalogue) were divided into five $W 1$ magnitude bins $(W 1<13,13 \leq W 1<14,14 \leq W 1<15,15 \leq W 1<16$, and $16 \leq W 1<17$ ); (2) five SVM algorithms separately tuned for these bins were used to classify galaxies, stars, and quasars; and (3) five SVM outputs were merged and treated as one final output. We verified that there is no evidence for an inconsistency between different $W 1$ magnitude bins.

Figure 2 shows normalized $W 1$ magnitude distributions for the three types of sources in the WISE $\times$ SCOS $\times$ SDSS crossmatch. As was shown in Kurcz et al. (2016), the derived classification statistics depends on the number of training objects, but the classifier stabilizes for subsamples of 3000 objects in each class. In our case, however, at both the bright $(W 1<13)$ and the faint end $(W 1>16)$, we did not have large enough numbers of sources to select randomly 3000 objects of each class from the input sets to build the training sample. In particular, we needed to use all the stars and quasars from these bins for the training and tests. For this reason, our training samples consist of different numbers of objects in each $W 1$ bin: 1000, 4000, 4000, 5000, and 2600 of each type for the $12<W 1<13,13<W 1<14$, $14<W 1<15,15<W 1<16$, and $16<W 1<17$ mag bins, respectively.

The first step of the tests was to determine the optimal $C$ and $\gamma$ parameters for the five $W 1$ bins, therefore we tuned five 


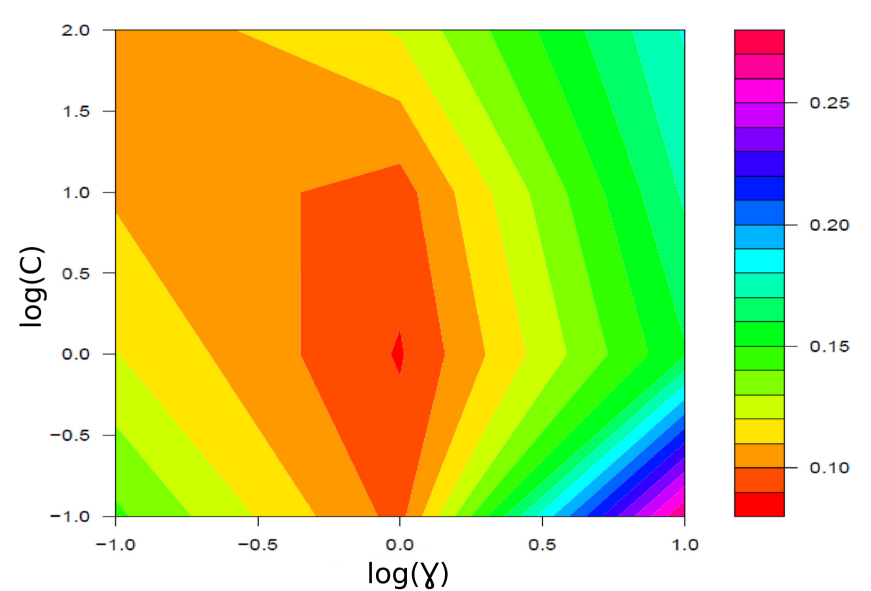

Fig. 3. Example of the $C-\gamma$ plane obtained from one of the five WISE $\times$ SCOS C-SVM classifiers. The mean misclassification rate (colour bar) as a function of the $C$ and $\gamma$ parameters was estimated through ten-fold cross-validation for each pair of the two parameters. The lower the misclassification rate, the better the performance of the SVM algorithm.

different C-SVM classifiers for our purpose. Figure 3 illustrates an exemplary grid search for one of the classifiers. The colours code the mean misclassification rate for given combinations of $\gamma$ and $C$; the lower the rate, the better the performance of the SVM algorithm. Here the misclassification rate is defined for each magnitude bin as the complement to the total accuracy (TA), the latter being the mean of accuracies $A_{i}$ for individual validation iterations:

$\mathrm{TA}=\frac{1}{10} \sum_{i=1}^{10} A_{i}$.

The accuracy for a given iteration is defined as

$A_{i}=\frac{\mathrm{TG}+\mathrm{TQ}+\mathrm{TS}}{\mathrm{TG}+\mathrm{TQ}+\mathrm{TS}+\mathrm{FG}+\mathrm{FQ}+\mathrm{FS}}$.

The components of this equation are true galaxies (TG), quasars (TQ) and stars (TS) from the training sample, properly classified as galaxies, quasars, and stars, respectively; and false galaxies (FG), which are real quasars or stars misclassified as galaxies, with false quasars (FQ) and false stars (FS) defined in a similar manner.

To further compare the performance of different classifiers, we calculated the following measures, as defined by Soumagnac et al. (2015): completeness $(c)$, contamination $(f)$, and purity $(p)$ for galaxy, star, and quasar samples. We used the following equations (here for galaxies):

$c_{\mathrm{g}}=\frac{\mathrm{TG}}{\mathrm{TG}+\mathrm{FGS}+\mathrm{FGQ}}$,

$f_{\mathrm{g}}=\frac{\mathrm{FSG}+\mathrm{FQG}}{\mathrm{TG}+\mathrm{FSG}+\mathrm{FQG}}$,

$p_{\mathrm{g}}=1-f_{\mathrm{g}}=\frac{\mathrm{TG}}{\mathrm{TG}+\mathrm{FSG}+\mathrm{FQG}}$,

where FGS and FGQ stand for galaxies misclassified as stars and quasars, and FSG, FQG are stars and quasars misclassified as galaxies. Definitions for stars and quasars follow in an analogous way. The accuracy for an individual class of objects is defined in the same way as the purity.
Table 1. Comparison of the performance for two classifiers: one using five parameters $(W 1, W 1-W 2, R-W 1, B-R$, w1mag13) and the other adding $W 3$ as the sixth parameter.

\begin{tabular}{c|cc|cc}
\hline \hline & \multicolumn{2}{|c|}{ 5D classifier } & \multicolumn{2}{c}{ 6D classifier } \\
& $c[\%]$ & $p[\%]$ & $c[\%]$ & $p[\%]$ \\
\hline Galaxies & 90.3 & 89.9 & 96.8 & 96.7 \\
Quasars & 95.1 & 92.2 & 98.1 & 98.6 \\
Stars & 90.0 & 88.5 & 96.9 & 98.1 \\
\hline TA & \multicolumn{2}{|c|}{$91.8 \%$} & \multicolumn{2}{c}{$97.3 \%$} \\
\hline
\end{tabular}

Notes. TA $=$ total accuracy; $c=$ completeness, and $p=$ purity, all calculated as a weighted arithmetic mean for all five $W 1$ bins.

\subsection{Usefulness of the $W 3$ passband for the classification}

We tested two classifiers for the separation between galaxies, quasars, and stars: one with five and the other with six parameters. These were $W 1$ magnitude, $W 1-W 2$ colour, $R-W 1$ colour, $B-R$ colour, and the w1mag13 differential aperture magnitude for the $W 1$ channel. The sixth parameter in the tests was the W3 magnitude, which is often used in WISE-based source classifications (e.g. Kovács \& Szapudi 2015; Ferraro et al. 2015), following the considerations of Wright et al. (2010), for example, that different types of sources occupy different regions of the $W 1-W 2$ vs. $W 2-W 3$ colour plane. However, as Fig. 1 shows, this idealized picture becomes more complicated for actual observations, and we decided to test how much the $W 3$ passband from WISE improves the automatic classification. We note that to avoid biases for overestimated fluxes, a recalibration of the W3 upper limits was necessary, as discussed in Sect. 2.1 and detailed in Appendix A; this did not prevent very low $\mathrm{S} / \mathrm{N}$ W3 measurements (which dominate the sample) from introducing possible confusion, however.

The results for the two classifiers are summarized in Table 1. The accuracy, completeness, and purity for both cases are very high. The contamination levels rarely exceed $10 \%$ and $5 \%$ for the 5D and 6D classifier, respectively. The 6D classifier clearly provides better results in all the calculated metrics, which shows that the availability of the $W 3$ band allows for (possibly considerable) improvement in the classification. However, the low detection rate in this band (only $30 \%$ of our sources have w3snr > 2) and the large variations in sensitivity on the $\mathrm{sky}^{6}$ mean that using this band might introduce biases into the final catalogue. Based on these considerations, together with the fact that each new classification parameter extends computation time, we decided not to use the $W 3$ passband for the final classification.

\subsection{General performance of the classifier}

To quantify the general performance of the set of five classifiers tuned for different $W 1$ magnitude bins, we analysed the final results (merged output catalogues from different $W 1$ bins) of the self-check and the test sample. As the test sample we randomly chose 5000 galaxies from the same WISE $\times$ SCOS $\times$ SDSScatalogue, independent of the training sample. The total accuracy, calculated over the galaxy test sample, was equal to $92.5 \%$. It was not possible to perform the same analysis for stars and quasars because for the brightest and faintest bins, all of them were used to build the training sample.

6 See e.g. http://wise2.ipac.caltech.edu/docs/release/ allsky/expsup/sec6_2.html 


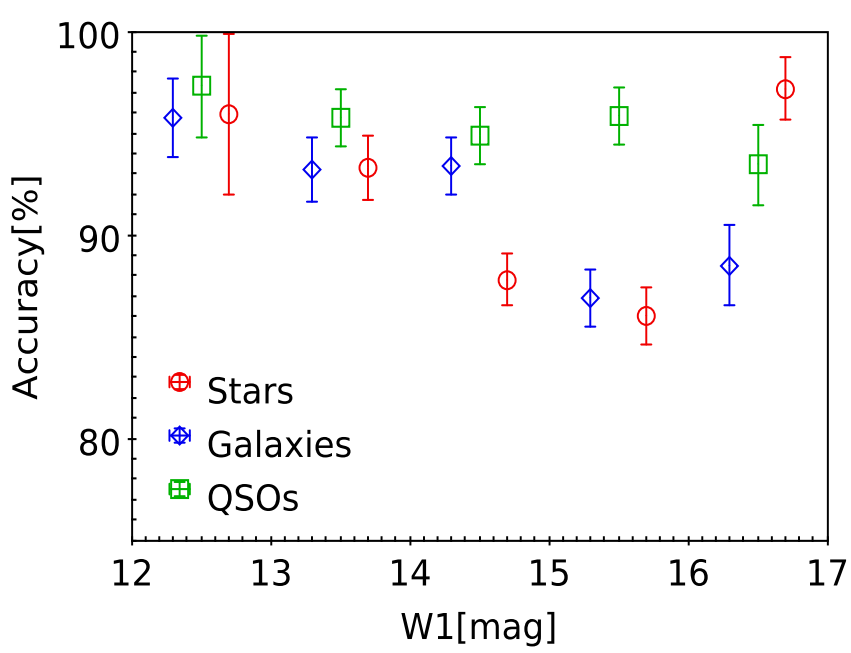

(a) Self-check

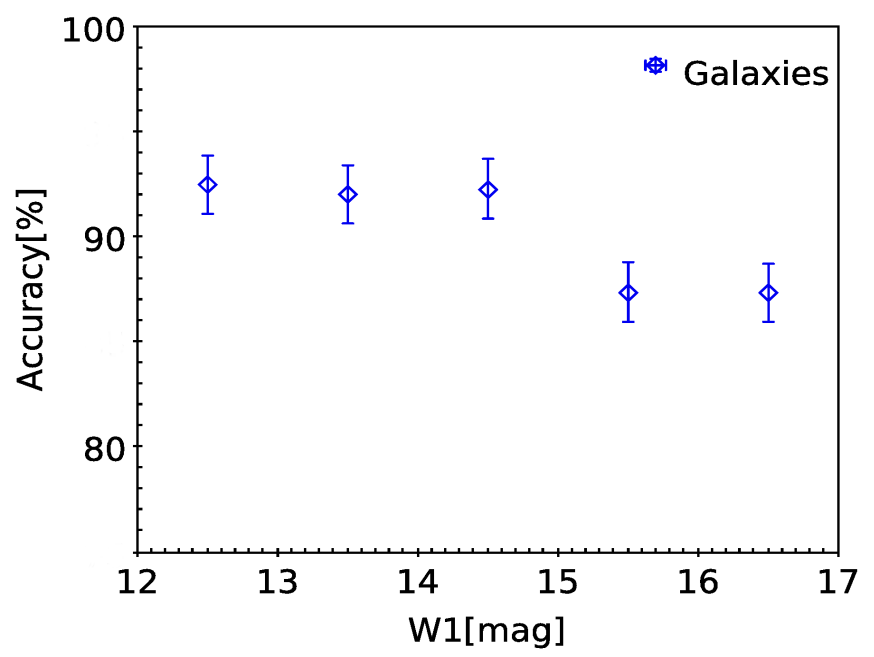

(b) Test sample

Fig. 4. Accuracy of the 5D classifier as a function of the limiting $W 1$ magnitude for the three types of classified sources, for the self-check (left panel) and the test sample (right panel). Blue diamonds correspond to galaxies, green squares to quasars, and red circles to stars. The points were shifted horizontally for clarity.

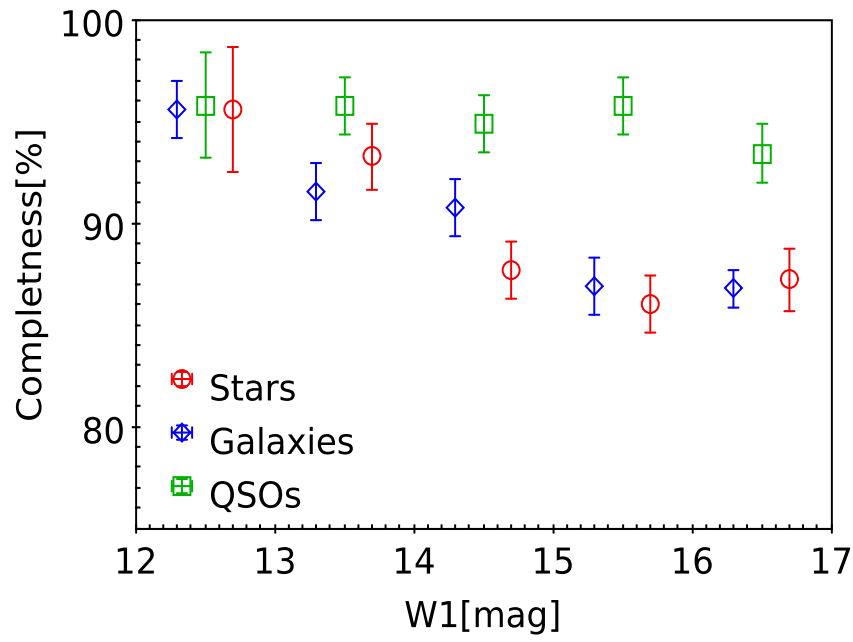

(a) completeness

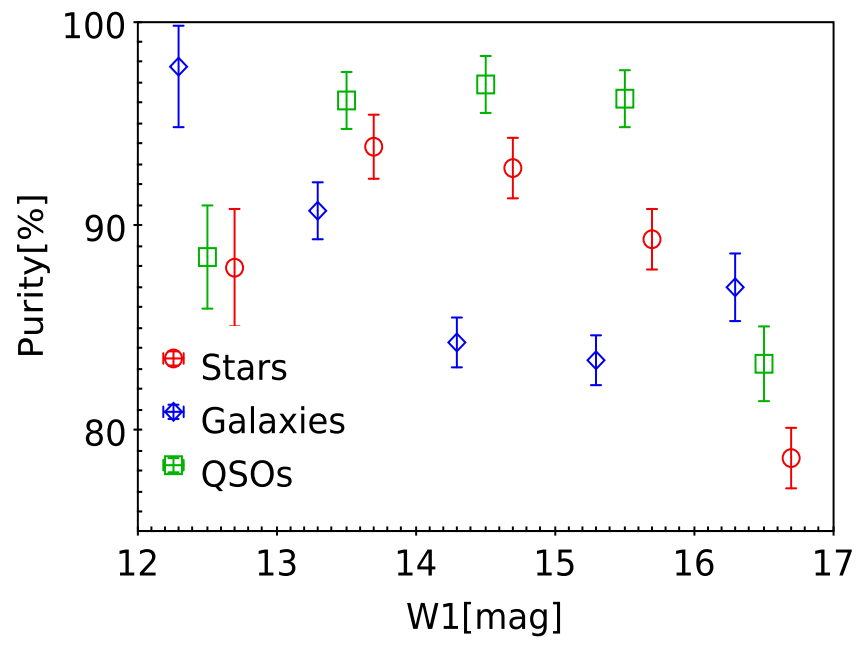

(b) purity

Fig. 5. Completeness and purity for galaxies, stars, and quasars as a function of the $W 1$ magnitude for the 5D classifier. These results refer to the self-check.

\subsubsection{Dependence on the $W 1$ magnitude}

After determining the total accuracy of the 5D classifier, we investigated its performance in more detail, starting with the dependence on the $W 1$ magnitude for the three classes. The results are illustrated in Fig. 4 for the self-check (left panel) and test samples (right panel, only for galaxies). In general the accuracies retain very high levels of about $90 \%$, but there is significant deterioration in classification quality for faint galaxies and stars. This is related to the fact that beyond $W 1 \gtrsim 15.5$ the training set contains very few galaxies and stars. The misclassification of galaxies occurs mainly for objects with $W 1>15 \mathrm{mag}$, and in most cases, true galaxies are misclassified as stars. The accuracy for galaxies calculated for the test sample has the same dependence on $W 1$ magnitude as the one derived from the self-check.

As we show in Fig. 5a, the completeness of the galaxy sample also decreases with increased $W 1$. For galaxies in the $15 \leq W 1<17 \mathrm{mag}$ bin, the completeness equals $\sim 87 \%$. This deterioration was expected, as there are far fewer training objects in the galaxy and star samples for the faintest $W 1$ bin than in the quasar sample.

We also checked the purity as a function of $W 1$ for the sources classified with the 5D classifier. As Fig. 5b shows, it is at similar levels as the completeness, although its dependence on $W 1$ is somewhat different. In particular, for all the three classes, there is a significant decrease in purity at the faint end. Still, as far as galaxies are concerned (of main interest for the present analysis), it stays at a reasonable level of $p>80 \%$ in all the bins.

Based on the findings of this section, we conclude that the $5 \mathrm{D}$ classifier is stable and can be safely used for the final classification. In principle, using the self-check and test results, we could estimate the main statistics of the final WISE $\times$ SCOS galaxy catalogue. The caveat is, however, that the SDSS training sample may not be representative enough for the WISE $\times$ SCOS dataset, which can lead to biases in such assessments of the final sample quality. 


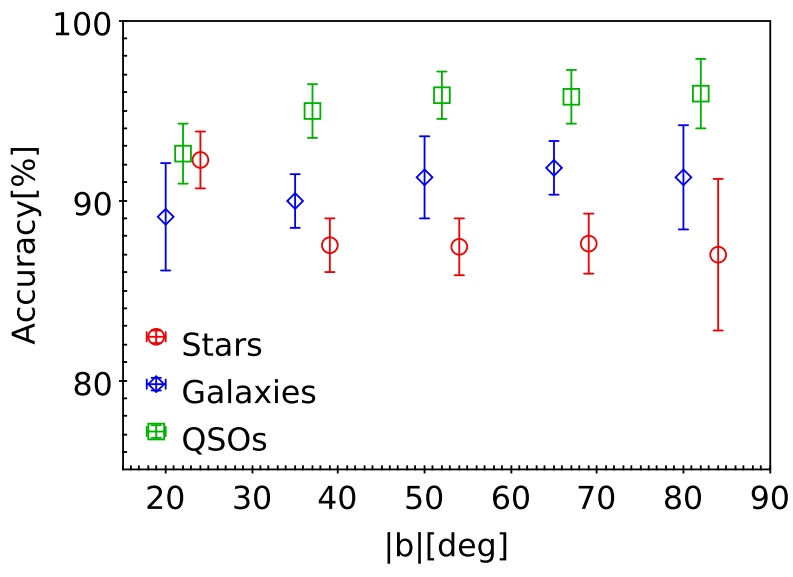

Fig. 6. Accuracy of the 5D classifier as a function of Galactic latitude for the three source classes.

In the final catalogue we will keep all the sources preselected as in Sect. 2, but owing to the above considerations, it might be preferable for more sophisticated analyses to remove the faintest sources to avoid possible misclassification. Nevertheless, we stress that the classification accuracy for the fainter part of our galaxy sample is still satisfying as it reaches very high levels even for the faintest sources $(87 \%$ for $15<W 1<16$ mag bin, and $\sim 90 \%$ for galaxies with $W 1>16$ mag for the self-check of the 5D classifier, and more than $85 \%$ for the galaxy test sample with $W 1>15 \mathrm{mag}$ ).

\subsubsection{Dependence on Galactic latitude}

We also checked how the accuracy of the five classifiers depends on Galactic latitude, $b$. We divided the training sample into six $15^{\circ}$-wide bins in $|b|$, and calculated the accuracy for each of the bins. The results are shown in Fig. 6. For the lowest latitude bin of $|b|<15^{\circ}$, the training sample contains practically no galaxies nor quasars, it was therefore not used in this test. This also means that to avoid extrapolation, this area may need to be discarded from the eventual galaxy catalogue.

\section{Results: final galaxy catalogue}

After thoroughly verifying the performance of the SVM algorithm on the test data, we applied it to the full WISE $\times$ SCOS sample described in Sect. 2. To prepare the final galaxy catalogue based on our automatic classification, we used additional information provided by SVM, namely the probabilities that the sources belong to particular classes. We also checked the catalogue for outliers in magnitude and colour space, and finally we compared it with the catalogue presented in B16, where simple colour cuts were employed to remove stars and quasars.

Although the SVM classifier assigns the final distinction based on discrete classes, it also provides additional information on object distance from different boundaries, which can be used as a probability for a given source to belong to a particular class. The probability calculated in SVM is given by the formula from Platt (1999), and this a posteriori probability function was implemented in the SVM kernel by Lin et al. (2007). For classification into more than two source types, the single class probabilities are combined together to estimate final probabilities by the pairwise coupling method (for detailed information see Wu et al. 2003). As our aim is to obtain a pure galaxy sample (with a strong decision value), we decided to take advantage of the full probability distributions to eliminate sources of unclear classification (located between different classes), instead of using discrete classes alone. Initially, the galaxy candidate catalogue output by SVM (i.e. such that $p_{\text {gal }}>p_{\text {star }}$ and $p_{\text {gal }}>p_{\mathrm{QSO}}$ ) included over 16.8 million sources. As in Kurcz et al. (2016), here we also checked whether cuts on source type probabilities might lead to an improvement in quality of the catalogue. Unlike in that analysis, however, in the case of WISE $\times$ SCOS galaxies even a cut of $p_{\text {gal }}>0.5$ (as well as more aggressive cuts) did not lead to an increase in the purity of the sample, while it lowered its completeness. For the subsequent analysis we therefore kept all the sources flagged as galaxies by SVM. We note that the derived SVM probability values are made available in the WISE $\times$ SCOS database. This will allow users to apply their own cuts to purify the sample (at the expense of completeness), for instance by setting maximum thresholds on $p_{\text {star }}$ and $p_{\mathrm{QSO}}$, or cutting more aggressively on $p_{\text {gal }}$.

The resulting catalogue was examined further for possible outliers in magnitude and colour space. Here we used the WISE $\times$ SCOS $\times$ SDSS data as the calibration to determine cases of extreme extrapolation from the training data. We found a very small number (only $\sim 1500$ ) of sources that had colours very different from those in the calibration sample. Most of them are located near the Galactic Plane or by the Magellanic Clouds, where WISE $\times$ SCOS photometry is problematic because of blending. These areas need to be masked out with a mask such as the one derived in B16. Applying that mask to the current catalogue, we were left with 15 million sources that are shown in Fig. 7.

\subsection{Comparison with Bilicki et al. (2016)}

It is interesting to compare the WISE $\times$ SCOS galaxy catalogue derived in this paper with the dataset presented in B16. The parent sample used in that work was the same as ours, but galaxies were separated from stars and quasars through colour cuts. In particular, the star-galaxy separation was made through a position-dependent cut in the $W 1-W 2$ colour to accommodate variations in the stellar locus with the position in the Galaxy. At high Galactic latitudes the cut was $W 1-W 2>0$, while it was gradually increased at lower latitudes to reach $W 1-W 2>0.12$ by the Galactic Plane and Bulge; see Sect. 4.2 and the appendix of B16 for details. To this, three cuts were added to mitigate stellar contamination and blending: (i) removal of the bright end of the sample $(W 1<13.8)$, which is dominated by stars on the one hand and is already sampled extragalactically by the 2MASS Photometric Redshift catalogue (2MPZ, Bilicki et al. 2014) on the other; (ii) a cutout of the Galactic Bulge reaching up to $|b|=$ $17^{\circ}$ at $\ell=0^{\circ}$; and (iii) manual cutouts of the Magellanic Clouds and M 31. Finally, quasars and blends thereof were removed by B16 with colour cuts in the $(W 1-W 2)-(R-W 2)$ plane: anything with $R-W 2>7.6-4(W 1-W 2)$ or $W 1-W 2>0.9$ was discarded. These cuts resulted in a dataset of 21.5 million sources; however, the sample still presented some spurious over- and underdensities in some areas, and an iterative procedure was performed to design the final mask. After the masking, the eventual WISE $\times$ SCOS galaxy catalogue of B16 included 18.7 million sources over $68 \%$ of the sky.

A cross-match of the SVM galaxy dataset with the dataset presented by B16 gives over 14.8 million common sources, which means that there are almost 2 million objects identified by SVM as galaxies that had been removed from the B16 sample. However, for this comparison to be meaningful, we should also remove the $W 1<13.8$ sources from the SVM catalogue, as well as those in the Bulge area, in the same way as in B16. 


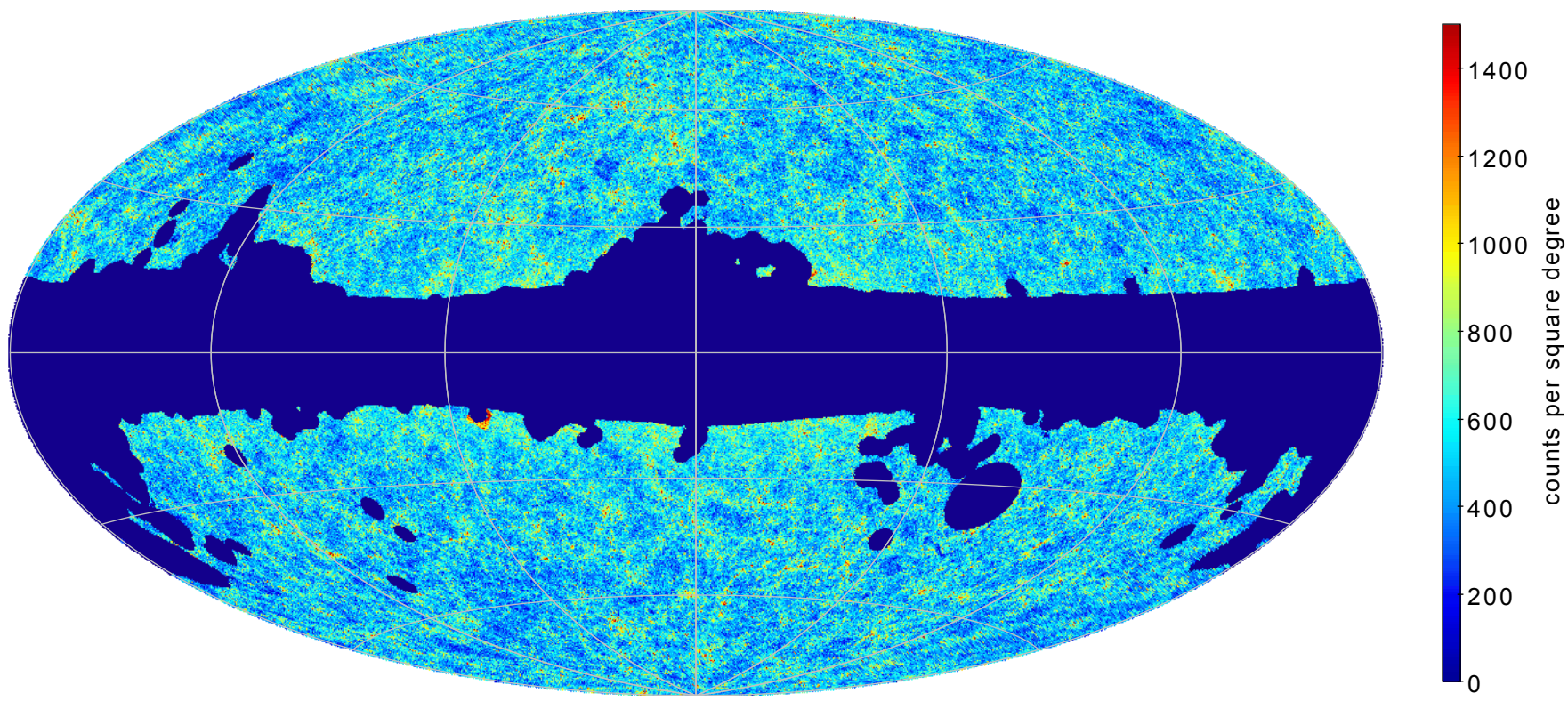

Fig. 7. Aitoff projection of sources identified by SVM as galaxy candidates in the WISE $\times$ SCOS catalogue after masking (see text for details). This plot shows 15 million objects in Galactic coordinates (with $\ell=0^{\circ}, b=0^{\circ}$ at the centre).

These two cuts reduce the sample generated by SVM but not by the colour cuts to 1.3 million, which is roughly $8 \%$ of the original SVM galaxy dataset. These objects are mostly concentrated at low Galactic latitudes $\left(|b|<30^{\circ}\right)$ and around the Magellanic Clouds, that is to say, in areas where the stellar blending that affects both parent catalogues has a negative impact on the photometry of extracted sources. Practically all of these objects have $W 1-W 2<0.12$, as expected (the upper limit of the B16 adaptive cut), and 1 million of them are outside the B16 mask. In general, the colours of the sources that are identified by SVM as galaxies but are absent from the B16 catalogue are consistent with those of SDSS stars or quasars.

Interestingly, practically no sources identified by B16 as quasars are present in the SVM galaxy catalogue: 1300 objects that meet the QSO colour criteria mentioned above are found in the SVM dataset. As those colour cuts were calibrated on a comparison of SDSS QSOs and GAMA galaxies (Liske et al. 2015), we conclude that our present catalogue is practically free of quasar contamination. This is consistent with the results from the tests presented in Sect. 4.2.

There are significantly more sources (5.6 million after masking) in the B16 galaxy catalogue that are absent from the SVM catalogue than the other way round. They are generally distributed over the entire sky, although their surface density increases towards the Galactic Plane. Their $W 1-W 2$ colour distribution is bimodal, with one peak at $W 1-W 2 \sim 0.1$ and the other at $W 1-W 2 \sim 0.5$. The former might indeed be stars that survived the position-dependent cut of B16, but were correctly classified by SVM. The latter are probably starburst or dusty galaxies, which our SDSS-based training sample is less sensitive to, hence they were partly misidentified by the classifier and removed from the SVM dataset; they were (correctly) kept in the B16 sample, however.

Finally, a comparison of source counts as a function of Galactic latitude (Fig. 8) suggests that the SVM catalogue is purer than the catalogue assembled by B16, as the rise in the number counts with decreasing absolute latitude occurs at lower $|b|$ in the former than in the latter. However, as B16 estimated that their catalogue was less than $90 \%$ complete at $|b|>30^{\circ}$

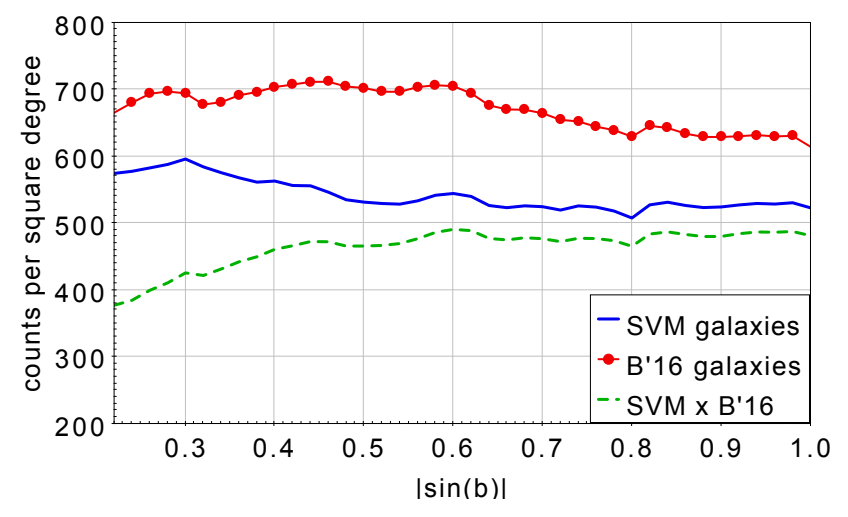

Fig. 8. Number counts as a function of the sine of Galactic latitude for three samples of WISE $\times$ SuperCOSMOS galaxies: selected with colour cuts by (Bilicki et al. 2016; red dotted), identified by SVM (present work; blue solid), and common to both datasets (green dashed).

( $|\sin b|>0.5)$, and the absolute counts of the present dataset are lower than those of the B16 sample even in the Galactic caps, we conclude that the higher purity of the SVM catalogue comes at the price of lower completeness.

\section{Summary}

The WISE $\times$ SCOS galaxy sample is currently the largest in terms of its size and sky coverage at $z \sim 0.2$, giving access to angular scales not accessible with samples such as SDSS. At the same time, it is much deeper than other all-sky datasets that are available from IRAS or 2MASS. Here we presented an approach to identify galaxies in the WISE $\times$ SCOS photometric data that is an alternative to the colour cuts applied in Bilicki et al. (2016). By using the support vector machines algorithm, trained and tested on a cross-match of spectroscopic SDSS data with WISE $\times$ SCOS, we identified about 15 million galaxy candidates over $70 \%$ of sky. This number is smaller than 18.5 million obtained by B16, mostly because our sample is of higher purity but lower completeness than the colour-selected sample. The resulting source probabilities assigned by SVM are 
provided in the photometric redshift WISE $\times$ SCOS dataset released together with the publication of $\mathrm{B} 16^{7}$.

We focused on galaxies because we used only extended (resolved) sources from SuperCOSMOS. Still, this work might be continued to obtain a more general identification of stars, galaxies, and quasars in the full WISE $\times$ SCOS sample. This would require SCOS point-source photometry to be calibrated all-sky in a similar way as the aperture-based measurements (Peacock et al. 2016), however, which currently is not the case.

Successful machine-learning galaxy identification in WISE $\times$ SCOS shows that a similar approach will be worthwhile for other samples based on WISE, cross-matched with forthcoming wide-angle datasets such as Pan-STARRS, SkyMapper, or VHS. For WISE itself, first efforts of all-sky star, galaxy, and QSO separation in that catalogue have been reported in Kurcz et al. (2016).

Acknowledgements. We are grateful to John Peacock for his useful comments Special thanks to Mark Taylor for the TOPCAT ${ }^{8}$ (Taylor 2005) and STILTS $^{9}$ (Taylor 2006) software. Some of the results in this paper have been derived using the HEALPix package ${ }^{10}$ (Górski et al. 2005). This publication makes use of data products from the Wide-field Infrared Survey Explorer, which is a joint project of the University of California, Los Angeles, and the Jet Propulsion Laboratory/California Institute of Technology, and NEOWISE, which is a project of the Jet Propulsion Laboratory/California Institute of Technology. WISE and NEOWISE are funded by the National Aeronautics and Space Administration. This research has made use of data obtained from the SuperCOSMOS Science Archive, prepared and hosted by the Wide Field Astronomy Unit, Institute for Astronomy, University of Edinburgh, which is funded by the UK Science and Technology Facilities Council. Funding for SDSS-III has been provided by the Alfred P. Sloan Foundation, the Participating Institutions, the National Science Foundation, and the US Department of Energy Office of Science. The SDSS-III web site is http://www.sdss3.org/. SDSS-III is managed by the Astrophysical Research Consortium for the Participating Institutions of the SDSS-III Collaboration including the University of Arizona the Brazilian Participation Group, Brookhaven National Laboratory, Carnegie Mellon University, University of Florida, the French Participation Group, the German Participation Group, Harvard University, the Instituto de Astrofisica de Canarias, the Michigan State/Notre Dame/JINA Participation Group, Johns Hopkins University, Lawrence Berkeley National Laboratory, Max Planck Institute for Astrophysics, Max Planck Institute for Extraterrestrial Physics, New Mexico State University, New York University, Ohio State University, Pennsylvania State University, University of Portsmouth, Princeton University, the Spanish Participation Group, University of Tokyo, University of Utah, Vanderbilt University, University of Virginia, University of Washington, and Yale University. M.B., K.M., A.P., A.K. and M.K. were supported by the Polish National Science Center under contract \#UMO-2012/07/D/ST9/02785. T.K., K.M. and A.P. were supported by the National Science Centre (grants UMO-2012/07/B/ST9/04425 and UMO-2013/09/D/ST9/04030), the Polish-Swiss Astro Project (co-financed by a grant from Switzerland, through the Swiss Contribution to the enlarged European Union), and the European Associated Laboratory Astrophysics PolandFrance HECOLS. M.B. was supported by the Netherlands Organization for Scientific Research, NWO, through grant No. 614.001.451, and through FP7 grant No. 279396 from the European Research Council.

\section{References}

Alam, S., Albareti, F. D., Allende Prieto, C., et al. 2015, ApJS, 219, 12 Assef, R. J., Stern, D., Kochanek, C. S., et al. 2013, ApJ, 772, 26
Beaumont, C. N., Williams, J. P., \& Goodman, A. A. 2011, ApJ, 741, 14 Bilicki, M., Jarrett, T. H., Peacock, J. A., Cluver, M. E., \& Steward, L. 2014, ApJS, 210, 9

Bilicki, M., Peacock, J. A., Jarrett, T. H., et al. 2016, ApJS, 225, 5

Bolton, A. S., Schlegel, D. J., Aubourg, E., et al. 2012, AJ, 144, 144

Brescia, M., Cavuoti, S., \& Longo, G. 2015, MNRAS, 450, 3893

Chang, C.-C., \& Lin, C.-J. 2011, ACM Transactions on Intelligent Systems and Technology, 2, 27:1-27:27, http://www.csie.ntu.edu.tw/cjlin/ libsvm

Cluver, M. E., Jarrett, T. H., Hopkins, A. M., et al. 2014, ApJ, 782, 90

Cristianini, N., \& Shawe-Taylor, J. 2000, An Introduction to Support Vector Machines: And Other Kernel-Based Learning Methods (Cambridge University Press)

Cutri, R. M., Wright, E. L., Conrow, T., et al. 2012, Explanatory Supplement to the WISE All-Sky Data Release Products, Tech. Rep.

Cutri, R. M., Wright, E. L., Conrow, T., et al. 2013, Explanatory Supplement to the AllWISE Data Release Products, Tech. Rep.

Ferraro, S., Sherwin, B. D., \& Spergel, D. N. 2015, Phys. Rev. D, 91, 083533

Górski, K. M., Hivon, E., Banday, A. J., et al. 2005, ApJ, 622, 759

Hambly, N. C., Davenhall, A. C., Irwin, M. J., \& MacGillivray, H. T. 2001a, MNRAS, 326, 1315

Hambly, N. C., Irwin, M. J., \& MacGillivray, H. T. 2001b, MNRAS, 326, 1295

Hambly, N. C., MacGillivray, H. T., Read, M. A., et al. 2001c, MNRAS, 326, 1279

Han, B., Ding, H.-P., Zhang, Y.-X., \& Zhao, Y.-H. 2016, Res. Astron. Astrophys., 16,005

Huertas-Company, M., Rouan, D., Tasca, L., Soucail, G., \& Le Fèvre, O. 2008, A\&A, 478, 971

Jarrett, T. H., Cohen, M., Masci, F., et al. 2011, ApJ, 735, 112

Jarrett, T. H., Cluver, M. E., Magoulas, C., et al. 2016, ArXiv e-prints [arXiv: 1607.01190]

Kovács, A., \& Szapudi, I. 2015, MNRAS, 448, 1305

Kurcz, A., Bilicki, M., Solarz, A., et al. 2016, A\&A, 592, A25

Lang, D., Hogg, D. W., \& Schlegel, D. J. 2016, AJ, 151, 36

Lin, H.-T., Lin, C.-J., \& Weng, R. C. 2007, Mach. Learn., 68, 267

Liske, J., Baldry, I. K., Driver, S. P., et al. 2015, MNRAS, 452, 2087

Manning, C. D., Raghavan, P., \& Schütze, H. 2008, Introduction to Information

Retrieval (New York, NY, USA: Cambridge University Press)

Marton, G., Tóth, L. V., Paladini, R., et al. 2016, MNRAS, 458, 3479

Małek, K., Solarz, A., Pollo, A., et al. 2013, A\&A, 557, A16

Meyer, D. 2001, R News, 1, 23

Peacock, J. A., Hambly, N. C., Bilicki, M., et al. 2016, MNRAS, 462, 2085

Platt, J. C. 1999, in Advances in Large Margin Classifiers (MIT Press), 61

Rahman, M., Ménard, B., \& Scranton, R. 2016, MNRAS, 457, 3912

Saglia, R. P., Tonry, J. L., Bender, R., et al. 2012, ApJ, 746, 128

Schlafly, E. F. \& Finkbeiner, D. P. 2011, ApJ, 737, 103

Schlegel, D. J., Finkbeiner, D. P., \& Davis, M. 1998, ApJ, 500, 525

Shawe-Taylor, J., \& Cristianini, N. 2004, Kernel Methods for Pattern Analysis (Cambridge University Press)

Skrutskie, M. F., Cutri, R. M., Stiening, R., et al. 2006, AJ, 131, 1163

Solarz, A., Pollo, A., Takeuchi, T. T., et al. 2012, A\&A, 541, A50

Soumagnac, M. T., Abdalla, F. B., Lahav, O., et al. 2015, MNRAS, 450, 666

Stern, D., Assef, R. J., Benford, D. J., et al. 2012, ApJ, 753, 30

Stoughton, C., Lupton, R. H., Bernardi, M., et al. 2002, AJ, 123, 485

Taylor, M. B. 2005, in Astronomical Data Analysis Software and Systems XIV, eds. P. Shopbell, M. Britton, \& R. Ebert, ASP Conf. Ser., 347, 29

Taylor, M. B. 2006, in Astronomical Data Analysis Software and Systems XV, eds. C. Gabriel, C. Arviset, D. Ponz, \& S. Enrique, Asp Conf. Ser., 351, 666 Vasconcellos, E. C., de Carvalho, R. R., Gal, R. R., et al. 2011, AJ, 141, 189

Woźniak, P. R., Williams, S. J., Vestrand, W. T., \& Gupta, V. 2004, AJ, 128, 2965 Wright, E. L., Eisenhardt, P. R. M., Mainzer, A. K., et al. 2010, AJ, 140, 1868 Wright, A. H., Robotham, A. S. G., Bourne, N., et al. 2016, MNRAS, 460, 765

Wu, T.-F., Lin, C.-J., \& Weng, R. C. 2003, J. Machine Learning Res., 5, 975

Yan, L., Donoso, E., Tsai, C.-W., et al. 2013, AJ, 145, 55

\footnotetext{
7 Available from the Wide Field Astronomy Unit, Institute for Astronomy, Edinburgh at http://ssa.roe.ac.uk/WISExSCOS.html

8 http://www.star.bristol.ac.uk/ mbt/topcat/

9 http://www.star.bristol.ac.uk/ mbt/stilts/

10 http://healpix. sourceforge.net/
} 


\section{Appendix A: Calibration of W3 and W4 upper limits}

The AllWISE Source Catalogue lists only the sources that were detected with $S / N \geq 5$ in at least one of the four survey bands. On the other hand, whenever there was a $5 \sigma$ detection in any of the bands, all the other bands were also measured at the given position, which means that each of the catalogued sources has magnitudes listed for all the bands (except for some very rare cases of processing or instrumental artefacts). Because of the much higher sensitivities in the $W 1$ and $W 2$ bands than in the two other channels, the AllWISE catalogue is mostly $W 1$ selected. In the $W 3$ channel $(12 \mu \mathrm{m})$, most of the $w 1$ snr $\geq 5$ sources will have $w 3 s n r<2$. Such objects are provided in the database as upper limits (or non-detections if w3snr < 0) and their fluxes are systematically overestimated. To be able to use such measurements in the classification procedure, we have designed an empirical correction for $W 3$ upper limits and non-detections. Analysing the dependence of the mean $W 3$ magnitude value on the $W 3 \mathrm{~S} / \mathrm{N}$ estimate from the database, we have found that there is a roughly constant shift in w3mpro at the $\mathrm{w} 3 \mathrm{snr}=2$ threshold, amounting to $\sim 0.75 \mathrm{mag}$, which can therefore be removed by artificially dimming the low-w3snr sources. Figure A.1 illustrate this calibration procedure for a random sample of WISE sources: the left panel shows quantities taken directly from the database, while the right panel presents our " $\mathrm{w} 3 \mathrm{cal}$ " magnitude on the $y$-axis, obtained by adding 0.75 mag to the database w3mpro value. Obviously, at w3snr $<0$, the values are pure noise. A similar procedure can be applied to the $W 4(\sim 23 \mu \mathrm{m})$ band, where the relevant offset for $\mathrm{w} 4 \mathrm{snr}<2$ sources was found to be the same as in $W 3$. This is illustrated in Fig. A.2. In this case, most of the sources remain undetected at all (w4snr $<0)$; for this reason, we did not use this band in our work.

We note that a more appropriate way of estimating magnitudes for the $W 3$ and $W 4$ upper limits and non-detections would be to use information from another band(s) in which a given source is detected with $S / N>5$ (for instance from the SuperCOSMOS bands). This is the idea behind the aperturematched photometry, employed by GAMA (Wright et al. 2016) and the forced-photometry technique applied to 400 million WISE sources selected from the SDSS (Lang et al. 2016). This method, albeit certainly of great interest, is beyond the scope of the present work, however.
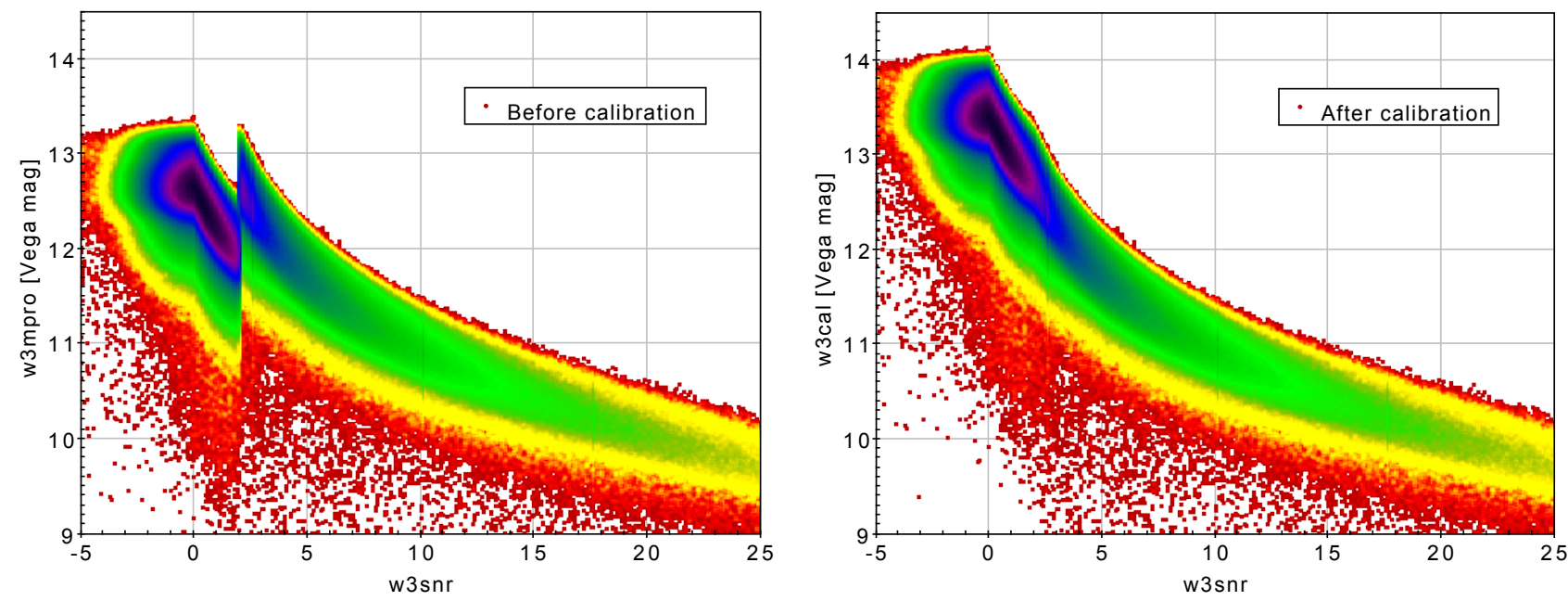

Fig. A.1. Illustration of the calibration procedure of $W 3$ upper limits and non-detections: values from the database (left panel) and after our empirical offset by +0.75 mag for the $\mathrm{w} 3 \mathrm{snr}<2$ sources (right panel).
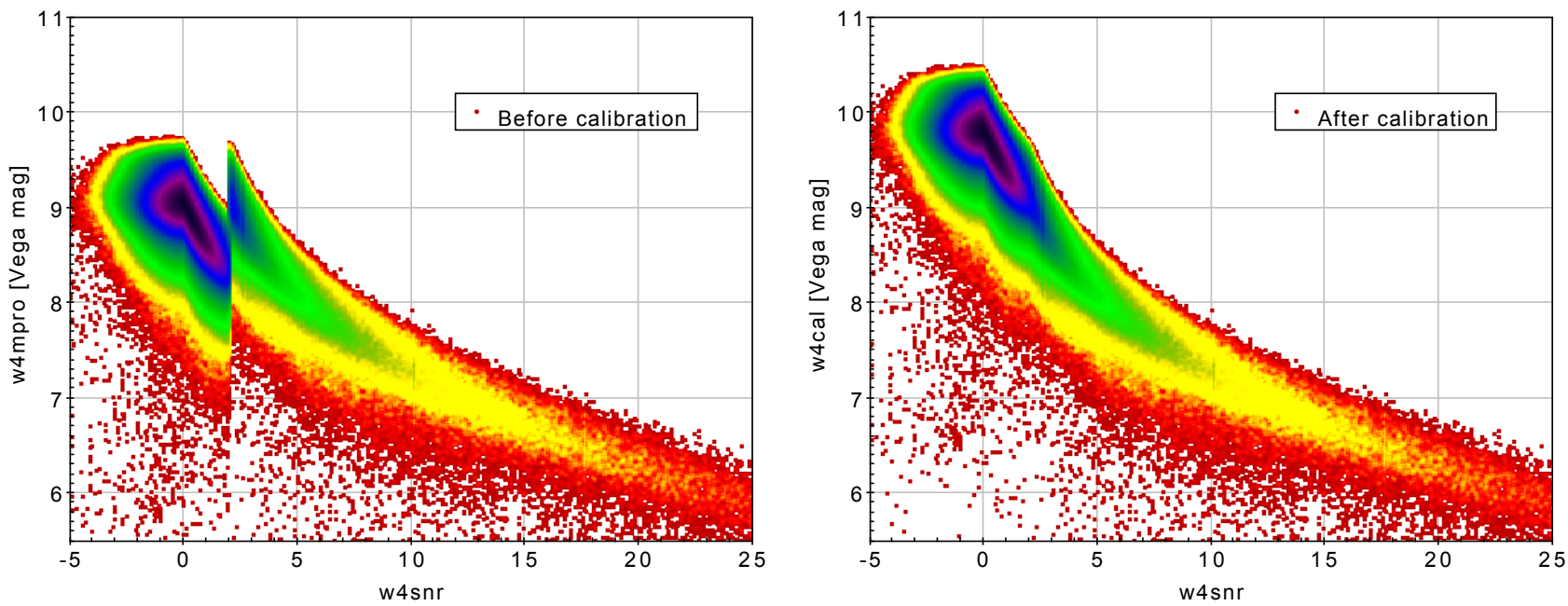

Fig. A.2. Illustration of the calibration procedure of $W 4$ upper limits and non-detections: values from the database (left panel) and after our empirical offset by +0.75 mag for the $\mathrm{w} 4 \mathrm{snr}<2$ sources (right panel). 